\title{
CMB dipole asymmetry through annular variance
}

\author{
M Valipour and M Farhang \\ Department of Physics, Shahid Beheshti University, Velenjak, Tehran, Iran \\ E-mail: m farhang@sbu.ac.ir
}

(Received 11 April 2019 ; in final form 17 September 2019)

\begin{abstract}
Dipole asymmetry is one of the most important anomalies in the cosmic microwave background (CMB). A dipole, if primordial, would challenge the isotropy of the Universe. In this work, we propose a novel method to find the direction of the dipole and its amplitude and assess its significance. The method is based on the comparison of annular variances on the sphere. We find the direction on the sphere around which the difference of the annular variances on the two hemispheres is maximized. By applying this algorithm on symmetric CMB simulations, we get the distribution of the measured dipole amplitude for these null cases, providing us with the baseline for quantifying the significance of the dipole amplitude of any other CMB maps. In particular, we find the statistical significance of the observed Planck dipole to be $1.6 \sigma$. Our simulations show that although the proposed method is not more powerful in detecting the dipole, as compared to other algorithms, its relatively low computational cost (performed in the pixel-space) can be regarded as its advantage. This paves the way for a straightforward upgrade of the method which uses spherical caps instead of rings; thus, by increasing the number of pixels used in calculating the variance, the results are improved significantly.
\end{abstract}

Keywords: CMB random field, statistical isotropy, variance

For full article, refer to the Persian section 